\title{
EDITORIAL
}

\section{Cathelicidin antimicrobial peptide as a serologic marker and potential pathogenic factor in antineutrophil cytoplasmic antibody-associated vasculitis}

\author{
Adil Gasim \\ See related research by Zhang et al., http://arthritis-research.com/content/15/5/R161
}

\begin{abstract}
Antineutrophil cytoplasmic antibodies are associated with pauci-immune small-vessel vasculitis and crescentic glomerulonephritis. Cathelicidin LL37 is the human member of a family of antimicrobial peptides that are released from activated neutrophils and monocytes at sites of acute inflammation. Zhang and colleagues evaluated serum levels of cathelicidin LL37 and interferon-alpha in patients with antineutrophil cytoplasmic antibody-associated vasculitis (AAV) and glomerulonephritis. Increased levels of cathelicidin LL37 and interferon-alpha were associated with AAV patients, particularly those with glomerular crescent formation. Cathelicidin LL37 may also be involved in the pathogenesis of AAV and thus could be a target for novel therapy. Cathelicidin LL37 is a promising new biomarker for active AAV, including aggressive crescentic glomerulonephritis, and may prove to be both a prognostic marker and a guide for treatment.
\end{abstract}

Zhang and colleagues have reported a potential role for the endogenous antimicrobial peptide cathelicidin LL37 and interferon-alpha (IFN $\alpha$ ) as serum markers for systemic and local inflammation in patients with antineutrophil cytoplasmic antibody-associated pauci-immune vasculitis and crescentic glomerulonephritis [1].

Cathelicidins are one family of antimicrobial peptides, with various immune functions. Cathelicidin LL37 (also known as human cationic antimicrobial protein, hCAP18)

Correspondence: husseing@email.unc.edu

Department of Pathology \& Laboratory Medicine, University of North Carolina at Chapel Hill, Chapel Hill, NC 27599-7525, USA is found within the lysosomes of macrophages and the secondary granules of neutrophils, and can be produced by epithelial cells $[1,2]$. Cathelicidin LL37 is an effector molecule of the innate immune system that is important in defense against microbial pathogens. Cathelicidins have also been proposed to play a role in the nonspecific defense against tumors [2], angiogenesis, and wound healing [3]. IFNo is a type I interferon that functions in antiviral, antiproliferative, and immunomodulatory activities.

Antineutrophil cytoplasmic antibody-associated vasculitis (AAV) is necrotizing vasculitis with few or no immune deposits that predominantly affects small vessels and is associated with antineutrophil cytoplasmic antibodies (ANCA) specific for myeloperoxidase (MPOANCA) or proteinase 3 (PR3-ANCA) [4]. MPO and PR3 are in the lysosomes of monocytes and the primary granules of neutrophils, and are released when neutrophils and monocytes are activated by ANCA $[5,6]$.

The role of cathelicidin LL37 in pathogenesis of different human diseases has been investigated by several research groups, mostly in the field of autoimmune and autoinflammatory diseases. Cathelicidin LL37 is overexpressed in inflamed skin in patients with psoriasis [7]. Human cathelicidin LL37 and its fragments inhibit HIV1 replication and the enzymes essential to the lifecycle of HIV, including HIV reverse transcriptase, HIV protease, and HIV integrase [8]. Plasma concentrations of cathelicidin LL37 are significantly higher in active hepatitis C virus infection and inactive hepatitis $\mathrm{B}$ virus infection, implicating cathelicidin LL37 in certain forms of hepatitis caused by hepatitis $C$ virus and hepatitis $B$ virus infections [9]. Human cathelicidins are involved in mucosal immunity of the respiratory tract, gastrointestinal tract, genitourinary tract, and eye [10]. Human 
cathelicidin is overexpressed in head and neck squamous cell carcinoma, and is strongly correlated with radiotherapy effectiveness [11].

Zhang and colleagues are the first to report elevated levels of cathelicidin LL37 in AAV, especially in patients with crescentic glomerulonephritis [1]. In this study, serum levels of cathelicidin LL37 and IFNa correlated with crescent formation and also with serum creatinine. This observation is important because the correlation with cellular crescents would indicate a correlation with disease activity, which could be an important prognostic factor and may even suggest novel strategies for treatment if the association results from a pathogenic role for cathelicidin LL37. This hypothesis would be further substantiated if the correlation is with active cellular crescents accompanied by necrosis rather than with fibrotic crescents accompanied by glomerular scarring. The presence of crescents, especially cellular crescents, is an important histological marker of active glomerulonephritis, which can be diagnosed only through renal biopsy, an expensive, time-consuming, and invasive technique. Cathelicidin LL37 is a serological marker, and is noninvasive and cost-effective compared with renal biopsy. Cathelicidin LL37 is stored as an inactive pro-form in secondary granules of neutrophils and is activated by PR3 (the autoantigen target of PR3-ANCA) when released at sites of inflammation [12]. Neutrophils and monocytes are activated by ANCA and release their lysosome and granule proteins, including MPO and PR3 $[5,6]$. Activated neutrophils also release cathelicidin LL37 [12,13]. Cathelicidin LL37 is found in neutrophil extracellular traps that are released by activated neutrophils [13]. These neutrophil extracellular traps have been implicated in the induction of autoimmunity and the mediation of inflammation in AAV [14].

To validate its value as a prognostic and predictive biomarker, future studies should investigate the levels of cathelicidin LL37 in patients with different severities of and different clinicopathologic phenotypes of AAV in large cohorts of patients with different levels of disease activity, remission, and relapse. More effective biomarkers are needed to improve prognostic and disease activity monitoring. Current clinical and laboratory observations that indicate a worse prognosis, especially for renal survival, are older age at diagnosis ( $>65$ years), more severe renal failure (especially dialysis dependency), and PR3-ANCA versus MPO-ANCA [15-18]. Patients with PR3-ANCA have higher mortality, a higher relapse rate, and more rapid renal deterioration compared with patients with MPO-ANCA [18]. In a given patient, however, there is no clear-cut value of ANCA levels using current clinical laboratory methods for predicting initial disease activity, induction of remission, or onset of relapse 18]. This situation may change with the development of more sophisticated assays for ANCA epitope specificity, but this has not been definitively documented in a clinical setting [19]. Pathologic evaluation of the diagnostic renal biopsy specimen provides some prognostic value [20], but is most effective for demonstrating advanced chronic disease that will not respond well to therapy [21]. One or more effective clinical laboratory tests for biomarkers that can predict outcome at the time of diagnosis, detect induction of remission, and identify relapses are badly needed to improve the management of ANCA disease. Cathelicidin LL37 and IFN $\alpha$ should be investigated further to determine whether they can serve this purpose.

In summary, the report by Ying Zhang and colleagues supports the possibility that cathelicidin LL37 and IFN $\alpha$ are useful serologic markers for active AAV, including aggressive crescentic glomerulonephritis. This study also raises the possibility that cathelicidin LL37 and IFNa could be involved in the pathogenesis of AAV and thus could be a target for novel therapy.

\section{Abbreviations}

AAV: Antineutrophil cytoplasmic antibody-associated vasculitis;

ANCA: Antineutrophil cytoplasmic antibodies; IFNa: Interferon-alpha MPO: Myeloperoxidase; PR3: Proteinase 3.

Competing interests

The author declares that he has no competing interests.

\section{Published: 28 Feb 2014}

\section{References}

1. Zhang Y, Shi W, Tang S, Li J, Yin S, Gao X, Wang L, Zou L, Zhao J, Huang Y, Shan L, Gounni AS, Wu Y, Yuan F, Zhang J: The influence of cathelicidin LL37 in human anti-neutrophils cytoplasmic antibody (ANCA)-associated vasculitis. Arthritis Res Ther 2013, 15:R161.

2. Heilborn JD, Nilsson MF, Chamorro Jimenez Cl, Sandstedt B, Borregaard N, Tham E, Sørensen OE, Weber G, Ståhle M: Antimicrobial protein hCAP18/ LL-37 is highly expressed in breast cancer and is a putative growth factor for epithelial cells. Int J Cancer 2005, 114:713-719.

3. Kittaka M, Shiba H, Kajiya M, Ouhara K, Takeda K, Kanbara K, Fujita T, Kawaguchi $\mathrm{H}$, Komatsuzawa $\mathrm{H}$, Kurihara $\mathrm{H}$ : Antimicrobial peptide LL37 promotes vascular endothelial growth factor-a expression in human periodontal ligament cells. J Periodontal Res 2013, 48:228-234.

4. Jennette JC, Falk RJ, Bacon PA, Basu N, Cid MC, Ferrario F, Flores-Suarez LF, Gross WL, Guillevin L, Hagen EC, Hoffman GS, Jayne DR, Kallenberg CGM, Lamprecht P, Langford CA, Luqmani RA, Mahr AD, Matteson EL, Merkel PA Ozen S, Pusey CD, Rasmussen N, Rees AJ, Scott DGl, Specks U, Stone JH, Takahashi K, Watts RA: Revised international Chapel Hill consensus conference nomenclature of vasculitides. Arthritis Rheum 2012, 2013:1-11.

5. Jennette JC, Falk RJ, Gasim AH: Pathogenesis of antineutrophil cytoplasmic autoantibody vasculitis. Curr Opin Nephrol Hypertens 2011, 20:263-270.

6. Jennette JC, Xiao H, Falk R, Gasim AM: Experimental models of vasculitis and glomerulonephritis induced by antineutrophil cytoplasmic autoantibodies. Contrib Nephrol 2011, 169:211-220.

7. Dombrowski Y, Schauber J: Cathelicidin LL-37: a defense molecule with a potential role in psoriasis pathogenesis. Exp Dermatol 2012, 21:327-330.

8. Wong JH, Legowska A, Rolka K, Ng TB, Hui M, Cho CH, Lam WW, Au SW, Gu OW, Wan DC: Effects of cathelicidin and its fragments on three key enzymes of HIV-1. Peptides 2011, 32:1117-1122.

9. lacob SA, Panaitescu E, lacob DG, Cojocaru M: The human cathelicidin LL37 peptide has high plasma levels in $B$ and $C$ hepatitis related to viral activity but not to 25-hydroxyvitamin D plasma level. Rom J Intern Med 2012, 50:217-223. 
10. Doss M, White MR, Tecle T, Hartshorn KL: Human defensins and LL-37 in mucosal immunity. J Leukocyte Biol 2010, 87:79-92.

11. Gilla K, Mohantib BK, Singha AK, Mishraa B, Deya S: The over expression of cathelicidin peptide LL37 in head and neck squamous cell carcinoma: the peptide marker for the prognosis of cancer. Cancer Biomarkers 2012, 10:125-134.

12. Jann NJ, Schmaler M, Kristian SA, Radek KA, Gallo RL, Nizet V, Peschel A, Landmann R: Neutrophil antimicrobial defense against Staphylococcus aureus is mediated by phagolysosomal but not extracellular trapassociated cathelicidin. J Leukoc Biol 2009, 86:1159-1169.

13. Van Avondt K, Fritsch-Stork R, Derksen RH, Meyaard L: Ligation of signal inhibitory receptor on leukocytes-1 suppresses the release of neutrophil extracellular traps in systemic lupus erythematosus. PLoS One 2013, 8:e78459.

14. Kessenbrock K, Krumbholz M, Schönermarck U, Back W, Gross WL, Werb Z, Gröne HJ, Brinkmann V, Jenne DE: Netting neutrophils in autoimmune small-vessel vasculitis. Nat Med 2009, 15:623-625.

15. Kaplan-Pavlovcic S, Cerk K, Kveder R, Lindic J, Vizjak A: Clinical prognostic factors of renal outcome in anti-neutrophil cytoplasmic autoantibody (ANCA)-associated glomerulonephritis in elderly patients. Nephrol Dial Transplant 2003, 18:v5-v7.

16. Slot MC, Tervaert JW, Franssen CF, Stegeman CA: Renal survival and prognostic factors in patients with PR3-ANCA associated vasculitis with renal involvement. Kidney Int 2003, 63:670-677.

17. Lionaki S, Blyth ER, Hogan SL, Hu Y, Senior BA, Jennette CE, Nachman PH, Jennette JC, Falk RJ: Classification of antineutrophil cytoplasmic autoantibody vasculitides: the role of antineutrophil cytoplasmic autoantibody specificity for myeloperoxidase or proteinase 3 in disease recognition and prognosis. Arthritis Rheum 2012, 64:3452-3462.

18. Specks U: Controversies in ANCA testing. Cleve Clin J Med 2012, 79:S7-S11.

19. Roth AJ, Ooi JD, Hess JJ, van Timmeren MM, Berg EA, Poulton CE, McGregor J, Burkart M, Hogan SL, Hu Y, Winnik W, Nachman PH, Stegeman CA, Niles J, Heeringa P, Kitching AR, Holdsworth S, Jennette JC, Preston GA, Falk RJ: Epitope specificity determines pathogenicity and detectability in ANCA-associated vasculitis. J Clin Invest 2013, 123:1773-1783.

20. Berden AE, Ferrario F, Hagen EC, Jayne DR, Jennette JC, Joh K, Neumann I, Noël LH, Pusey CD, Waldherr R, Bruijn JA, Bajema IM: Histopathologic classification of ANCA-associated glomerulonephritis. J Am Soc Nephrol 2010, 21:1628-1636.

21. Ford SL, Polkinghorne KR, Longano A, Dowling J, Dayan S, Kerr PG, Holdsworth SR, Kitching AR, Summers SA: Histopathologic and clinical predictors of kidney outcomes in ANCA-associated vasculitis. Am J Kidney Dis 2014, 63:227-235.

\subsection{6/ar4495}

Cite this article as: Gasim: Cathelicidin antimicrobial peptide as a serologic marker and potential pathogenic factor in antineutrophil cytoplasmic antibody-associated vasculitis. Arthritis Research \& Therapy 2014, 16:105 